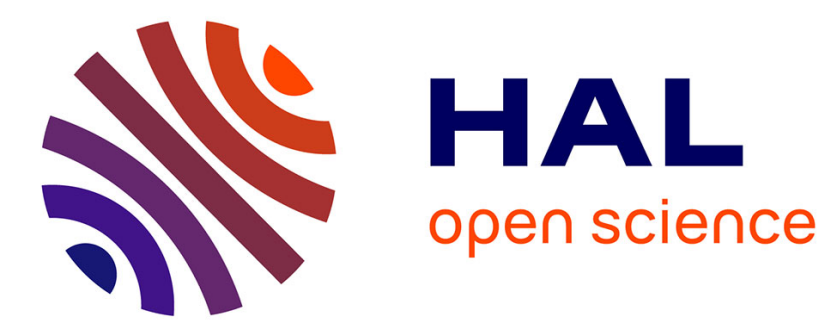

\title{
Efficient series expansion for Matrix inversion with application to MMSE equalization
}

\author{
Nicolas Le Josse, Christophe Laot, Karine Amis Cavalec
}

\section{To cite this version:}

Nicolas Le Josse, Christophe Laot, Karine Amis Cavalec. Efficient series expansion for Matrix inversion with application to MMSE equalization. IEEE Communications Letters, 2008, 12 (1), pp.35 - 37. 10.1109/LCOMM.2008.071274 . hal-01893758

\section{HAL Id: hal-01893758 https://hal.science/hal-01893758}

Submitted on 11 Jun 2021

HAL is a multi-disciplinary open access archive for the deposit and dissemination of scientific research documents, whether they are published or not. The documents may come from teaching and research institutions in France or abroad, or from public or private research centers.
L'archive ouverte pluridisciplinaire HAL, est destinée au dépôt et à la diffusion de documents scientifiques de niveau recherche, publiés ou non, émanant des établissements d'enseignement et de recherche français ou étrangers, des laboratoires publics ou privés. 


\title{
Efficient series expansion for matrix inversion with application to MMSE equalization
}

\author{
Nicolas Le Josse, Christophe Laot and Karine Amis \\ GET/ENST Bretagne, Signal and Communication Dept./TAMCIC (CNRS UMR 2872) \\ Technopôle Brest-Iroise, CS 83818, 29238 Brest Cedex 3, France \\ Email:\{nicolas.lejosse;christophe.laot;karine.amis\}@enst-bretagne.fr \\ Tel : 33 (0)2 290010 14, Fax: 33 (0)2 29001012
}

\begin{abstract}
In this paper, we describe an efficient approach to overcome the need for matrix inversion required in most wired applications encoutered in practice. In particular, MMSE equalization based on series expansion to approximate the matrix inversion is addressed. By adjusting a scaling factor, the series expansion is directly optimized according to a fixed order with respect to a system performance criterion. In comparison with previous approaches, the resulting equalizer enables improved BER performance according to a fixed order, in addition to low complexity without the need for a complicated eigenvalue calculation procedure.
\end{abstract}

Index Terms-Matrix inversion, series expansion, MMSE equalization, low complexity receiver.

\section{INTRODUCTION}

Equalization has proven to be an effective means for removing intersymbol interference (ISI). In particular, it has been demonstrated that a minimum mean square error (MMSE) equalizer can efficiently deal with this interference. Unfortunately, its computational cost is too high and makes its implementation difficult since it requires large matrix inversion [1]. To overcome the need for matrix inversion, the authors proposed in [2], [3] an approximate MMSE equalizer employing a finite series expansion that results from a truncated Taylor series expansion. The resulting detector requires the computation of only one scaling factor to ensure convergence. Unfortunately, for many systems, a high order is required to keep high performance at the expense of computational complexity. Alternatively, with the help of the Cayley-Hamilton theorem [1], matrix inversion can be expressed as a finite sum of a weighted matrix polynomials. The coefficients are chosen to optimize some performance measure at the equalizer output [4], [5]. However, the complexity involved in the weight optimization problem does not seem to be easier than performing a direct matrix inversion. Based on [2], [3], we propose to derive an optimal series expansion from a truncated Taylor series expansion according to a fixed order and a system performance criterion. In contrast with previous approaches, the resulting equalizer enables improved bit error rate (BER) performance according to a fixed order, in addition to low complexity without the need for a complicated procedure to calculate the eigenvalues.

This work was supported in part by FT RD.

\section{SYSTEM DESCRIPTION}

Consider the discrete-time baseband communication system in which modulated symbols are transmitted over an ISI channel corrupted by AWGN. We assume the following standard vector representation

$$
\mathbf{y}(d)=\mathbf{H s}(d)+\mathbf{b}(d)
$$

where $\mathbf{y}(d)=[y(d+k)]_{0 \leq k \leq N_{F}-1}$ is a vector of $N_{F}$ output symbols and $\mathbf{s}(d)=[s(d+\bar{k})]_{-(\Delta+1) \leq k \leq N_{F}+L-(\Delta+1)}$ (resp $\left.\mathbf{b}(d)=[b(d+k)]_{0 \leq k \leq N_{F}-1}\right)$ the corresponding vector of inputs (resp. noise) i.i.d, of zero-mean and variance $\sigma_{s}^{2}$ (resp. $\left.\sigma_{b}^{2}\right)$. $\mathbf{H}$ represents the $N_{F} \times\left(N_{F}+L-1\right)$ channel Toeplitz matrix of the corresponding discrete-time equivalent channel model $\{h(l)\}_{0 \leq l \leq L-1}$ of length $L$. The index $\Delta$ is the decision delay parameter required in the derivation of the equalizer transfer function. The output of the channel is processed by the sliding window MMSE filter at the receiver to further suppress ISI. It consists of a $N_{F}$ tap-weight vector $\mathbf{w}^{\mathrm{H}}$ which minimizes the cost function $\mathrm{E}\left[\left|\mathbf{w}^{\mathrm{H}} \mathbf{y}(d)-s(d)\right|^{2}\right]$ such that

$$
\mathbf{w}^{\mathrm{H}}=\sigma_{s}^{2} \mathbf{h}_{\Delta}^{\mathrm{H}} \boldsymbol{\Sigma}^{-1}
$$

in which $\boldsymbol{\Sigma}$ is the $N_{F} \times N_{F}$ covariance matrix of the received signal given by $\boldsymbol{\Sigma}=\sigma_{s}^{2} \mathbf{H} \mathbf{H}^{\mathrm{H}}+\sigma_{b}^{2} \mathrm{I}_{N_{F} \times N_{F}}$ and $\mathbf{h}_{\Delta}$ is the $\Delta^{t h}$ column vector of channel matrix $\mathbf{H}$ corresponding to the different fadings experienced by the desired component. Equalization requires a minimum filter length $N_{F}$ to achieve good performance [6] at the expense of high computational complexity mainly due to the calculation of $\boldsymbol{\Sigma}^{-1}$.

\section{EFFICIENT APPROXIMATE MMSE EQUALIZER BASED ON SERIES EXPANSION}

\section{A. Scenario}

Let $\lambda_{i}, i=1, \ldots, N_{F}$ denote the positive real eigenvalues of $\boldsymbol{\Sigma}$. Now consider a scaling factor $\psi \in \mathcal{R}$, which is determined for the matrix $\boldsymbol{\Sigma}$ such that $\left|1-\psi \lambda_{i}\right|<1, i=1, \ldots, N_{F}$. If the previous condition is satisfied, we can introduce the series expansion $\boldsymbol{\Sigma}^{-1}=\psi \sum_{i=0}^{\infty}\left(\mathbf{I}_{N_{F}}-\psi \boldsymbol{\Sigma}\right)^{i}$. By considering the $K$ th order series expansion in $\boldsymbol{\Sigma}$ to approximate the matrix inversion in (2), the corresponding equalizer is given by

$$
\begin{gathered}
\mathbf{w}_{(K)}^{\mathrm{H}}=\sigma_{s}^{2} \mathbf{h}_{\Delta}^{\mathrm{H}} \psi\left(\mathbf{I}_{N_{F}}+\left(\mathbf{I}_{N_{F}}-\psi \boldsymbol{\Sigma}\right)+\left(\mathbf{I}_{N_{F}}-\psi \boldsymbol{\Sigma}\right)^{2}+\ldots\right. \\
\left.+\left(\mathbf{I}_{N_{F}}-\psi \boldsymbol{\Sigma}\right)^{K}\right)
\end{gathered}
$$


which converges from the matched-filter $(K=0)$ detector to the MMSE equalizer $(K \rightarrow \infty)$, as the order $K$ grows from zero to infinity, i.e. $\lim _{K \rightarrow \infty} \mathbf{w}_{(K)}^{\mathrm{H}}=\mathbf{w}^{\mathrm{H}}$. From this point of view, it is advantageous for computational simplicity to keep $K$ as low as possible while still maintaining sufficient performance.

\section{B. Proposed approach}

Let $\lambda_{\min }$ and $\lambda_{\max }$ denote the smallest and the largest eigenvalues of $\boldsymbol{\Sigma}$. A simple analysis shows that the convergence of $\mathbf{w}_{(K)}^{\mathrm{H}}$ to $\mathbf{w}^{\mathrm{H}}$ as $K \rightarrow \infty$ is ensured by choosing $\psi$ in the range of $0<\psi<\frac{2}{\lambda_{\max }}$. In [2], the authors propose to set $\psi=\frac{2}{\operatorname{trace}(\boldsymbol{\Sigma})}$ since $\operatorname{trace}(\boldsymbol{\Sigma}) \geq \lambda_{\max }$. There is a possibility of estimating a more suitable scaling factor if the eigenvalues of $\boldsymbol{\Sigma}$ are known. The fastest convergence rate takes place when the two extreme modes $\left|1-\psi \lambda_{\min }\right|$ and $\left|1-\psi \lambda_{\max }\right|$ are identical such that

$$
\psi=\frac{2}{\lambda_{\min }+\lambda_{\max }}
$$

These results are well-known and have previously been presented [3]. However when the simulation context is associated with a large spread of eigenvalues, high order $K$ may be required to keep high performance, at the expense of computational complexity. Therefore, instead of ensuring that $\mathbf{w}_{(K)}^{\mathrm{H}}$ performs the fastest convergence rate to $\mathbf{w}^{\mathrm{H}}$ as $K$ grows from zero to infinity, we propose an alternative criterion to set $\psi$ such that $\mathbf{w}_{(K)}^{\mathrm{H}}$ achieves an optimum for some performance measure according to a fixed order $K$. In that sense, we denote $\psi(K)$ the new scaling factor. Based on this approach, we propose to derive an efficient approximate MMSE equalizer based on the first order series expansion of $\Sigma^{-1}$ that achieves the best signal interference to noise ratio (SINR) at the equalizer output. More explicitly, the first order approximate MMSE equalizer equation is given by

$$
\mathbf{w}_{(1)}^{\mathrm{H}}=\sigma_{s}^{2} \mathbf{h}_{\Delta}^{\mathrm{H}} \psi(1)\left(\mathbf{I}_{N_{F}}+\left(\mathbf{I}_{N_{F}}-\psi(1) \boldsymbol{\Sigma}\right)\right)
$$

By considering the expression of the SINR at the equalizer output, the optimum scaling factor $\psi(1)$ can then be found by solving $\left(\psi^{\mathrm{opt}}(1)\right)=\operatorname{argmax}_{(\psi(1))}\left(\frac{\left\|\mathbf{w}_{(1)}^{\mathrm{H}} \mathbf{h}_{\Delta}\right\|^{2}}{\mathbf{w}_{(1)}^{\mathrm{H}} \mathbf{\Sigma} \mathbf{w}_{(1)}}\right)$ under the constraint that $\psi(1)$ ensures the convergence of $\mathbf{w}_{(K)}^{\mathrm{H}}$ to $\mathbf{w}^{\mathrm{H}}$ as $K \rightarrow \infty$. The solution can be expressed as a function of the equalizer coefficients

$$
\psi(1)=2 \frac{\mathbf{v}^{\mathrm{H}} \boldsymbol{\Theta} \mathbf{v}}{\mathbf{v}^{\mathrm{H}} \boldsymbol{\Theta} \mathbf{\Sigma} \mathbf{v}}
$$

where we define $\mathbf{v}^{\mathrm{H}}=\mathbf{h}_{\Delta}^{\mathrm{H}} \boldsymbol{\Sigma}$ and $\boldsymbol{\Theta}=\left\|\mathbf{h}_{\Delta}\right\|^{2} \mathbf{I}_{N_{F}}-\mathbf{h}_{\Delta} \mathbf{h}_{\Delta}^{\mathrm{H}}$. More details on the derivation can be found in the appendix. The structure which implements the new equalizer is shown in Fig. 1. In comparison with previous approaches [2], [3], the proposed series expansion achieves the best SINR at the equalizer output according to a fixed order without the need for a complicated eigenvalue calculation procedure.

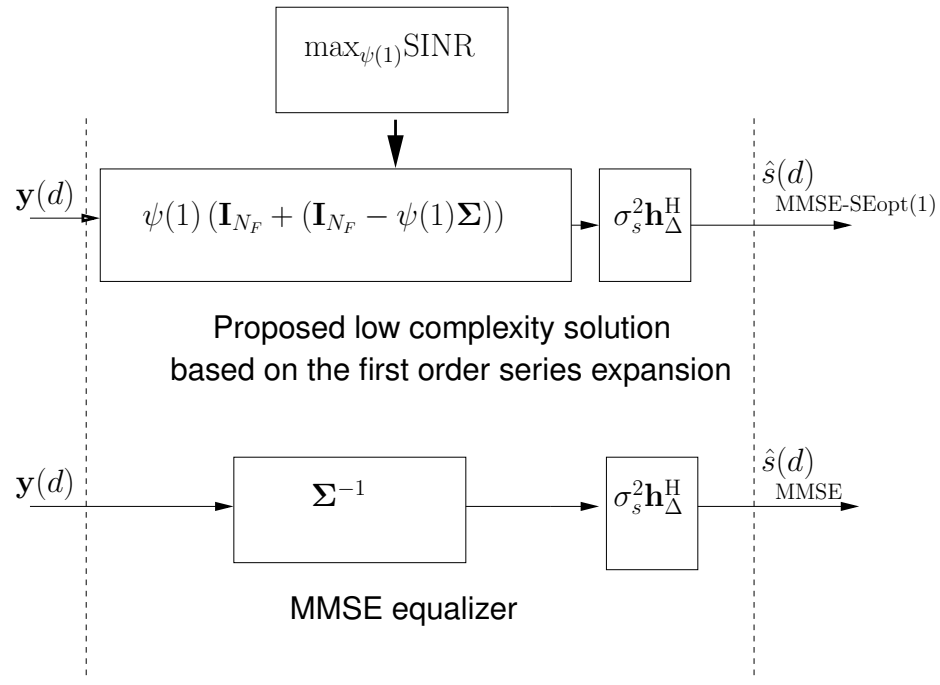

Fig. 1. First order approximate MMSE equalizer block diagram in comparison to that of the MMSE equalizer.

\section{Simulation RESUlts AND DisCUSSION}

Simulations consider a 4PSK baseband model where $\sigma_{s}^{2}=$ 1. The channel is time-invariant and given by the following set of real coefficients [0.5679 -0.11360 .58490 .11240 .556 ]. Simulations have shown that setting the filter coefficient number $N_{F}=21$ and the decision delay parameter $\Delta=10$ is sufficient to achieve good BER performance.

In the following, MMSE-SEopt $(\mathrm{K})$ (resp. MMSE-SEev $(K)$ ) denotes the $K$ th order approximate MMSE equalizer computed from (6) (resp. (4)). In Fig. 2, the BER after equalization is plotted as a function of $E_{b} / N_{0}$ for $K=0,1,8$ and $K \rightarrow \infty$. It should be noted that the complexity involved to obtain an exact expression of $\psi(8)$ becomes quite high and its value can be found using a numerical approach. This involves searching for the numerical value that maximizes the ratio $\frac{\left\|\mathbf{w}_{(8)}^{\mathrm{H}} \mathbf{h}_{\Delta}\right\|^{2}}{\mathbf{w}_{(8)}^{\mathrm{H}} \mathbf{\Sigma} \mathbf{w}_{(8)}}$ over a discrete set of values.

At low $\frac{E_{b}}{N_{0}}<10 d B$, the MMSE-SEopt(8) and MMSESEev(8) performance are very close to that of the MMSE$\operatorname{SEopt}(\infty)$ equalizer (MMSE equalizer), while taking $K=0$ (matched filter) causes a noticeable degradation. Interestingly, the MMSE-SEopt(1) performance enables a good compromise between the matched filter $(K=0)$ and the MMSE equalizer $(K \rightarrow \infty)$ with direct matrix inversion, i.e. complexity and ISI reduction. For $\frac{E_{b}}{N_{0}}=7 d B$, the MMSE-SEopt(1) BER performs better (resp. worse) by about a factor 2 (resp. 2) than the MMSE-SEopt(0) (resp. the MMSE-SEopt $(\infty)$ ). Compared to the MMSE-SEev(1) BER, that of the MMSE-SEopt(1) is improved by a factor 1.3. The MMSE-SEopt(1) equalizer is able to retrieve a large part of the ISI from the received signal and high performance can already be obtained. To compare the interest of the proposed method with that in [2], the 8th order approximate MMSE equalizer performance obtained from $\psi=\frac{2}{\operatorname{trace}(\boldsymbol{\Sigma})}$ (section (III-B)) is also reported (MMSESEtr(8)). Compared to the MMSE-SEopt(1) (resp. MMSESEopt(8)) BER, the MMSE-SEtr(8) BER leads to significant performance loss.

At high $\frac{E_{b}}{N_{0}}>10 \mathrm{~dB}$, we observe that the performance 
gap between the MMSE-SEopt $(K)$ for $K=0,1,8$ and the MMSE-SEopt $(\infty)$ increases. The ISI phenomenon dominates over the noise such that the equalizer has to invert the channel impulse response. As a consequence, a high order series expansion is necessary to keep high performance. Nevertheless, we notice that the MMSE-SEopt $(K)$ BER is still significantly improved compared to that of the $\operatorname{MMSE-SEev}(K)$ (resp. MMSE-SEtr $(K))$ for $K=1$ and $K=8$. For $\frac{E_{b}}{N_{0}}=17 d B$, the MMSE-SEopt(1) (resp. MMSE-SEopt(8)) BER performs better by about a factor 1.7 (resp. 2) from the MMSE-SEev(1) (resp. the MMSE-SEev(8)) BER.

Simulations over various frequency selective channels have shown that the same observations can be made, which confirms the relevance of the proposed approach.

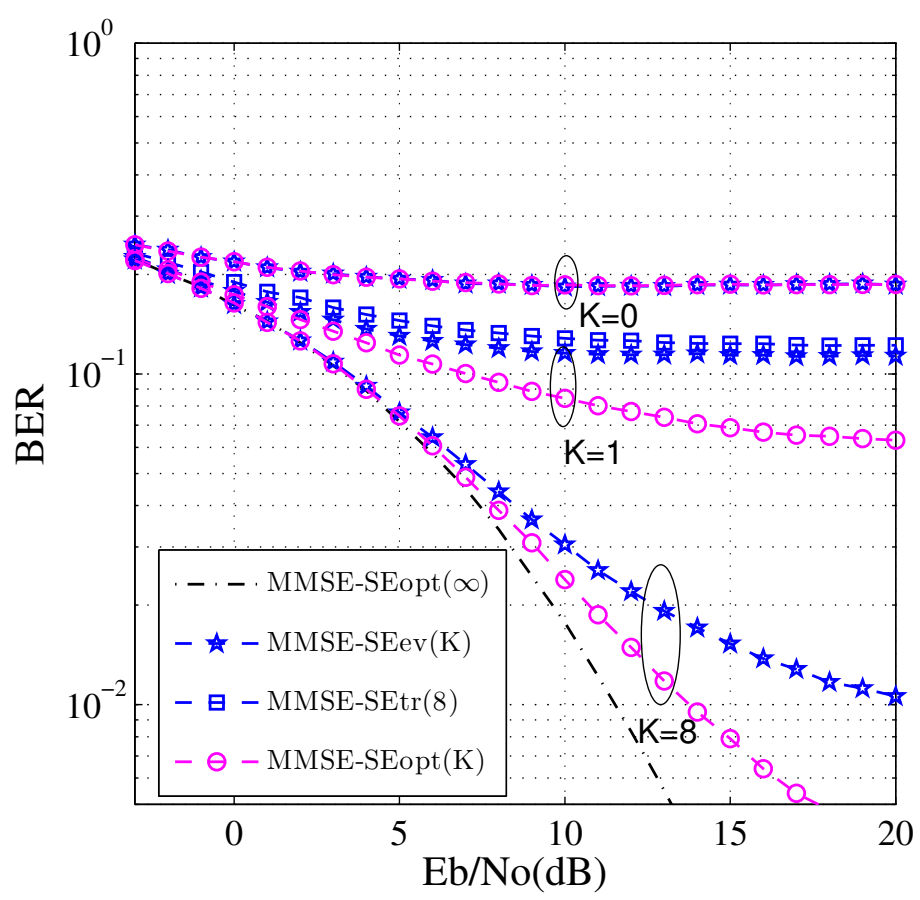

Fig. 2. BER performance comparison of the proposed $K$ th order approximate MMSE equalizer (MMSE-SEopt $(K))$ for $K=0,1,8$ and the corresponding MMSE equalizer with direct matrix inversion (MMSE-SEopt $(\infty)$ ) over frequency selective channel of order $L=5$ and 4PSK modulated signals.

\section{CONCLUSION}

This paper describes an efficient approach based on series expansion to overcome the need for the complicated matrix inversion required in MMSE equalization derivation. According to a fixed order, the proposed series expansion ensures the best performance at the equalizer output as well as lower complexity in comparison with previous approaches. The resulting approximate MMSE equalizer yields high performance even for a small order as demonstrated in our simulations. In addition, the corresponding complexity mainly depends on calculating $\psi(K)$. Future work will consider the computation cost reduction of $\psi(K)$.

\section{APPENDIX}

From (5), the output of the equalizer is given by

$$
\hat{s}(d)=\mathbf{w}_{(1)}^{\mathrm{H}} \mathbf{h}_{\Delta} s(d)+\mathbf{w}_{(1)}^{\mathrm{H}}\left(\mathbf{y}(d)-\mathbf{h}_{\Delta} s(d)\right)
$$

where the variance of the output of the equalizer and the useful signal are given by $\mathrm{E}\left\{|\hat{s}(d)|^{2}\right\}=\mathbf{w}_{(1)}^{\mathrm{H}} \boldsymbol{\Sigma} \mathbf{w}_{(1)}$ and $\mathrm{E}\left\{\left|\mathbf{w}_{(1)}^{\mathrm{H}} \mathbf{h}_{\Delta} s(d)\right|^{2}\right\}=\sigma_{s}^{2}\left\|\mathbf{w}_{(1)}^{\mathrm{H}} \mathbf{h}_{\Delta}\right\|^{2}$ respectively. The corresponding SINR is defined as

$$
\begin{aligned}
\operatorname{SINR} & =\frac{\sigma_{s}^{2}\left\|\mathbf{w}_{(1)}^{\mathrm{H}} \mathbf{h}_{\Delta}\right\|^{2}}{\mathbf{w}_{(1)}^{\mathrm{H}} \mathbf{\Sigma} \mathbf{w}_{(1)}-\sigma_{s}^{2}\left\|\mathbf{w}_{(1)}^{\mathrm{H}} \mathbf{h}_{\Delta}\right\|^{2}} \\
& =\left(\frac{\mathbf{w}_{(1)}^{\mathrm{H}} \mathbf{\Sigma} \mathbf{w}_{(1)}}{\sigma_{s}^{2}\left\|\mathbf{w}_{(1)}^{\mathrm{H}} \mathbf{h}_{\Delta}\right\|^{2}}-1\right)^{-1}
\end{aligned}
$$

where $\frac{\mathbf{w}_{(1)}^{\mathrm{H}} \boldsymbol{\Sigma} \mathbf{w}_{(1)}}{\sigma_{s}^{2}\left\|\mathbf{w}_{(1)}^{\mathrm{H}} \mathbf{h}_{\Delta}\right\|^{2}}>1$. Then, maximizing SINR and the ratio $\frac{\sigma_{s}^{2}\left\|\mathbf{w}_{(1)}^{\mathrm{H}} \mathbf{h}_{\Delta}\right\|^{2}}{\mathbf{w}_{(1)}^{\mathrm{H}} \mathbf{\Sigma} \mathbf{w}_{(1)}}$ are equivalent. The optimum scaling factor $\psi(1)$ that achieves the best SINR at the equalizer output can be found by setting

$$
\frac{\mathrm{d}}{\mathrm{d} \psi(1)}\left(\frac{\left\|\mathbf{w}_{(1)}^{\mathrm{H}} \mathbf{h}_{\Delta}\right\|^{2}}{\mathbf{w}_{(1)}^{\mathrm{H}} \mathbf{\Sigma} \mathbf{w}_{(1)}}\right)=0
$$

Substituting (5) in (9) gives

$$
\frac{\mathrm{d}}{\mathrm{d} \psi(1)}\left(\frac{\left(2|| \mathbf{h}_{\Delta} \|^{2}-\psi(1) \mathbf{h}_{\Delta}^{\mathrm{H}} \boldsymbol{\Sigma} \mathbf{h}_{\Delta}\right)^{2}}{4 \mathbf{h}_{\Delta}^{\mathrm{H}} \boldsymbol{\Sigma} \mathbf{h}_{\Delta}-4 \psi(1) \mathbf{h}_{\Delta}^{\mathrm{H}} \boldsymbol{\Sigma}^{2} \mathbf{h}_{\Delta}+\psi(1)^{2} \mathbf{h}_{\Delta}^{\mathrm{H}} \boldsymbol{\Sigma}^{3} \mathbf{h}_{\Delta}}\right)=0
$$

So we deduce that the SINR will be maximum if

$$
\psi(1)=2 \frac{\underline{\mathbf{v}}^{\mathrm{H}} \underline{\boldsymbol{\Theta} \mathbf{v}}}{\underline{\mathbf{v}}^{\mathrm{H}} \underline{\boldsymbol{\Theta} \mathbf{\Sigma}}}
$$

where we define $\mathbf{v}^{\mathrm{H}}=\mathbf{h}_{\Delta}^{\mathrm{H}} \boldsymbol{\Sigma}$ and $\Theta=\left\|\mathbf{h}_{\Delta}\right\|^{2} \mathbf{I}_{N_{F}}-\mathbf{h}_{\Delta} \mathbf{h}_{\Delta}^{\mathrm{H}}$. It can be shown that the resulting $\psi(1)$ ensures the convergence of $\mathbf{w}_{(K)}^{\mathrm{H}}$ to $\mathbf{w}^{\mathrm{H}}$ as $K \rightarrow \infty$.

\section{REFERENCES}

[1] G. Golub and C. Van Loan, "Matrix Computations," John Hopkins Univ. Press, 1989.

[2] Z. Lei and T. Lim, "Simplified Polynomial-expansion Linear Detectors for DS-CDMA Receivers," IEEE Elec. Letters, vol. 34, no. 16, pp 15611563, 1998.

[3] R. T. M. Mozaffaripour, "Suboptimum Search Algorithm in Conjunction With Polynomial Expanded Multiuser Detection for Uplink," Wireless Personnal Comm., Kluwer Academic Publishers, vol. 24, no. 1, pp 1-9, 2003.

[4] S. Moshavi, E. Kanterakis, and D. Schilling, "Multistage linear receivers for DS-CDMA systems," Int. J. Wireless Inform. Networks, vol. 3, no. 1, pp 1-17, Jan. 1996.

[5] C. Boulanger and L. Ouvry, "Multistage linear DS-CDMA receivers," IEEE ISSSTA 98 Proc., Sun City, vol. 2, pp 663-667, 2-4 sept. 1998.

[6] K. Amis, N. Le Josse, and C. Laot, "Efficient Frequency-Domain MMSE turbo equalization derivation and performance comparison with the TimeDomain counterpart," Wireless and Mobile Comm., ICWMC 2007, March. 2007. 